\title{
The natural compound codonolactone attenuates TGF-ß1-mediated epithelial-to-mesenchymal transition and motility of breast cancer cells
}

\author{
JIANJIANG FU ${ }^{1}$, XIAOQIN KE ${ }^{1}$, SONGLIN TAN ${ }^{1}$, TING LIU ${ }^{1}$, SHAN WANG ${ }^{1}$, \\ JUNCHAO MA ${ }^{1}$ and HONG LU ${ }^{2}$ \\ ${ }^{1}$ Department of Pharmacology, School of Pharmacy, Jiangxi University of Traditional Chinese Medicine, \\ Nanchang, Jiangxi 330004; ${ }^{2}$ Network and Educational Technology Center, Jiangxi University of \\ Traditional Chinese Medicine, Nanchang, Jiangxi 330004, P.R. China
}

Received June 25, 2015; Accepted August 5, 2015

DOI: 10.3892/or.2015.4394

\begin{abstract}
Codonolactone (CLT), a natural product, is the major bioactive component of Atractylodes lancea, and also found in a range of other medical herbs, such as Codonopsis pilosula, Chloranthus henryi Hemsl and Atractylodes macrocephala Koidz. This sesquiterpene lactone has been demonstrated to exhibit a range of activities, including anti-allergic activity, anti-inflammatory, anticancer, gastroprotective and neuroprotective activity. Previously, we found that CLT showed significant anti-metastatic properties in vitro and in vivo. In order to determine whether EMT-involved mechanisms contribute to the anti-metastatic effects of CLT, we checked the anti-EMT properties of CLT and its potential mechanisms. Here it was demonstrated that CLT inhibited TGF- $\beta 1$-induced epithelial-mesenchymal transition (EMT) in vitro and in vivo. Furthermore, downregulation of TGF- $\beta$ signaling was associated with the anti-EMT properties of CLT. Data from western blotting showed that, in breast cancer cells, TGF- $\beta 1$ stimulated the activation of Runx 2 , and CLT blocked the activation of Runx2. Finally, to verify whether CLT-induced EMT inhibition leads to suppression of metastatic potential, the effects of CLT on cell invasion and migration were determined. It was found that TGF- $\beta 1-$ induced migration and invasion was significantly blocked by CLT in both MDA-MB-231 and MDA-MB-468 cells. Collectively, our findings demonstrated that CLT inhibited programming of EMT in vitro and in vivo, resulting in inhibition of motility of metastatic breast cancer cells. The
\end{abstract}

Correspondence to: Dr Hong Lu, Network and Educational Technology Center, Jiangxi University of Traditional Chinese Medicine, 818 Xingwan Road, Nanchang, Jiangxi 330004, P.R. China

E-mail: jianghong_6666@126.com

Key words: codonolactone, epithelial-to-mesenchymal transition, metastasis, TGF- $\beta$ signaling, breast cancer inhibitory effect of CLT was due to its ability to inhibit TGF- $\beta$ signaling and Runx 2 phosphorylation.

\section{Introduction}

For decades, advances in the breast cancer field have dramatically promoted the overall 5-year survival rate for women in the United States from $75 \%$ between 1975 to 1977 , to $90 \%$ between 2002 to 2008 (1). Despite these advances, breast cancer is still the second cause of death from cancer. Most of the patients died of metastases, rather than the primary tumors, and the 5-year survival rate is a mere $23 \%$ for the women diagnosed with distant metastatic disease (1). It was reported that metastasis to distant sites accounts for $>90 \%$ of breast cancer-related mortality (2). Despite its clinical importance, metastasis remains the most insidious aspect of breast cancer. Consequently, there are few successful treatments that directly target this stage of carcinogenesis. Therefore, unraveling the complexities of the genetic and biochemical determinants of metastasis and developing effective therapies are urgently needed to improve treatment regimens and ultimately prognostic outcomes.

A recent focus in breast cancer metastasis research is the epithelial-mesenchymal transition (EMT). Originally, EMT is reported to be a critical developmental program that entails epithelial cells to mesenchymal cells, mobiling cells and giving rise to bone, muscle, connective tissue and blood vessels (3). Increasing data have shown that EMT processes have been implicated in cancer progression and metastasis including carcinogenesis, increased motility, invasion, and angiogenesis in various solid tumor types (4). A wide spectrum of alterations occurs during EMT processes, promoting loss of cell-cell adhesion, leading to a shift in cytoskeletal dynamics and a change from epithelial morphology and physiology to the mesenchymal phenotype (5). Several oncogenic pathways that respond to extracellular cues have been shown to contribute to EMT of carcinoma cells, such as transforming growth factor- $\beta$ (TGF- $\beta$ ) and bone morphogenetic protein (BMP), Wnt/ $\beta$-catenin, Notch and Hedgehog signaling pathway. These pathways have activated transcription factors including Snail, 
Slug, and the Twist to generate many intermediate phenotypes between epithelial and mesenchymal states (6).

CLT, also named atractylenolide III, is the major bioactive component of Atractylodes lancea and also found in a range of medical herbs, such as Codonopsis pilosula, Atractylodes macrocephala Koidz and Chloranthus henryi Hemsl (7). This sesquiterpene lactone has been demonstrated to exhibit a range of activities, including anti-allergic activity $(8,9)$, anti-inflammatory (10), gastroprotective and neuroprotective activity $(11,12)$. Furthermore, CLT was shown recently to induce apoptosis of A549 cells via mitochondria-mediated death pathway (13). Previously, we focused our interest on antitumor activities of CLT, and revealed that this natural compound exhibited anti-metastatic effects in metastatic breast cancer cells, and these effects may be mediated by inhibition of matrix metalloproteinases (MMPs) via downregulation of the transcriptional activity of Runx2 (14). Runx2 is a key transcription factor associated with osteoblast differentiation and breast cancer metastasis. There is close association between abnormal activation of Runx 2 and EMT, matrix degradation, angiogenesis and colonizing in bone marrow (15). Moreover, this transcription factor is also the common target of TGF- $\beta$ / BMP signaling $(16,17)$. Based on their critical roles in TGF- $\beta$ signaling and Runx2 during EMT, we hypothesized that EMT-involved mechanisms may contribute to the anti-metastatic effects of CLT. Therefore, we evaluated the effects of CLT and its mechanisms on EMT processes in breast cancer.

\section{Materials and methods}

Materials. Codonolactone (CLT) with a purity of up to $98.0 \%$ was from Shanghai PureOne Biotechnology (P0110; Shanghai, China). A stock solution was used throughout the study by dissolving CLT in $100 \%$ dimethyl sulfoxide (DMSO). The final DMSO concentration did not exceed $0.1 \%$. The molecular structure of CLT is presented in Fig. 1. TGF- $\beta 1$ recombinant human protein (PHG9211) is the product of Life Technologies, and 5-fluorouracil (5-FU, cat no. PHR1227; Sigma-Aldrich, Beijing, China) was used as positive controls in in vivo study. Batimastat (Bat, cat no. SC-203833; Santa Cruz Biotechnology Inc., Santa Cruz, CA, USA) was used in in vitro invasion and migration assay and served as a positive control. This compound is a potent, broad spectrum MMP inhibitor, and exhibits anti-invasive and anti-metastatic activity.

Cell culture. Human breast cancer MDA-MB-468 and MDA-MB-231 cells, purchased from Cell Resource Center, Institute of Basic Medical Sciences, Chinese Academy of Medical Sciences, were maintained in Dulbecco's modified Eagle's medium (DMEM) supplemented with $10 \%$ fetal bovine serum (FBS; Gibco, Inc.), $100 \mathrm{IU} / \mathrm{ml}$ penicillin, and $100 \mu \mathrm{g} / \mathrm{ml}$ streptomycin (Invitrogen, Inc.) in a humidified incubator containing $5 \% \mathrm{CO}_{2}$ at $37^{\circ} \mathrm{C}$.

Animals and ethics statement. Five- to 6-week-old female NOD/SCID mice were purchased from Vital River Laboratories (Beijing, China) to establish an orthotopic xenograft tumor model. All animal studies were conducted in strict accordance with the requirements of animal experimental protocol, which was approved by the Animal Ethics Committees of Jiangxi
University of Traditional Chinese Medicines. All surgery was performed under sodium pentobarbital anesthesia, and all efforts were made to minimize suffering.

In vivo growth assay. For the growth assay, MDA-MB-468 cell xenografts were established by injection of $1 \times 10^{7}$ cells at the mammary fat pad in NOD/SCID mice. After 3 weeks of growth, the tumors were removed and chopped into $1 \times 1 \times 1-m m$ tumor pieces, and these pieces were then implanted into the mammary fat pad of mice. The mice bearing tumor chunks were randomly divided into 4 groups: control, 5-FU (100 mg/kg/week) treatment group, CLT (25 mg/kg/day) treatment group and CLT $(75 \mathrm{mg} / \mathrm{kg} /$ day $)$ treatment group. Forty-eight hours later, CLT and was administered by gavage on a regimen of 6-day dosing per week for 5 weeks. 5-FU was treated by intraperitoneal injection on a regimen of 3-day dosing per week for 5 weeks. Tumor growth was assessed by measuring the length and width of tumors with electronic calipers every 3-4 days continuously. Volumes were calculated using the formula: (length) $\mathrm{x}$ (width) ${ }^{2} / 2$.

Immunohistochemistry for tumor tissues. Cytokeratin 19 (CK19) and vimentin in tumor tissues were determined by immunostaining. Formalin-fixed, paraffin-embedded MDA-MB-468 tumor tissues were cut into $4-\mu$ m-thick sections. After dewaxing and hydration, the slides were incubated with proteinase $\mathrm{K}$ at $37^{\circ} \mathrm{C}$ for $15 \mathrm{~min}$ to retrieve antigen. Then the sections were treated with $3 \% \mathrm{H}_{2} \mathrm{O}_{2}$ in methanol for $10 \mathrm{~min}$. Followed by blocking with $10 \%$ normal goat serum (Cell Signaling Technology, Danvers, MA, USA), the slides were incubated with CK19 (1:50, ab15463; Abcam) or vimentin antibody (1:50, 3932; Cell Signaling Technology) or PBS $(0.01 \mathrm{~mol} / \mathrm{l})$ at $4^{\circ} \mathrm{C}$ overnight. The slices were incubated with second antibody (HRP-linked secondary antibody) at room temperature for $60 \mathrm{~min}$, and then peroxidase activity was detected by SignalStain ${ }^{\circledR}$ DAB Substrate kit (Cell Signaling Technology) or AEC Substrate system (ab64252; Abcam). All the slides were checked under light microscopy (BX-63; Olympus), and images were analyzed by Image Pro Plus software 5.0 (Media Cybernetics Inc., Silver Spring, MD, USA).

In vitro invasion assay. Effects of CLT on TGF- $\beta 1$-induced invasion of breast cancer cells were measured by a 48 -well microchemotaxis system (AP 48; Neuro Probe, Gaithersburg, MD, USA). Briefly, individual filters were coated with $5 \mu \mathrm{g}$ Matrigel and fibronectin (served as a chemoattractant) on the smooth (upper) and rough (lower) surface, respectively. Thirty microliters Medium (30 $\mu \mathrm{l})$ containing 0.1\% BSA was added into each lower chamber, and then the chambers were fixed. Cells $\left(5 \times 10^{5} /\right.$ well) in $100 \mu \mathrm{l} \mathrm{FBS-free} \mathrm{medium} \mathrm{containing}$ vehicle, Bat or CLT were added separately to the top of this Matrigel layer in the presence of $10 \mathrm{ng} / \mathrm{ml} \mathrm{TGF}-\beta 1$ (final concentration). After 14-h incubation, the filters were fixed with methanol and stained with $0.5 \%$ crystal violet for $60 \mathrm{~min}$. The cells on the upper surface of the filters were removed by wiping with cotton swabs. The cells invading to the lower surface of the filter through Matrigel and filter were quantified with Image Pro Plus software 5.0 (Media Cybernetics Inc.) and the most representative results are illustrated in the figures. Each assay was performed in triplicate. 


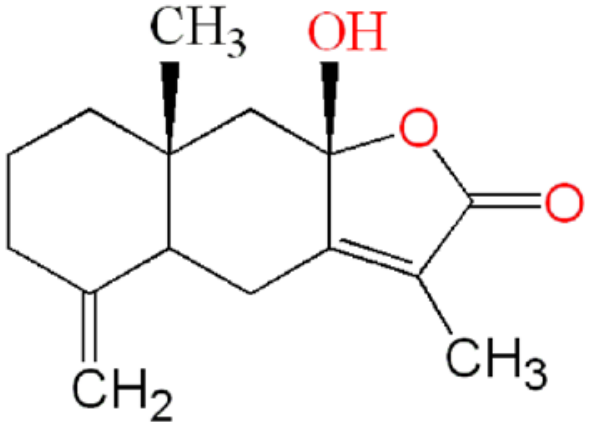

Codonolactone

Figure 1. Molecular structure of codonolactone.

In vitro migratory assay. In vitro migration of breast cancer cells induced by TGF- $\beta 1$ was measured using Oris ${ }^{\mathrm{TM}}$ Cell Migration Assay (Platypus Technologies, Madison, WI, USA) following the manufacturer's instructions. Log-phase cells were harvested, washed three times with serum-free medium, and resuspended into a final concentration of $4 \times 10^{6} / \mathrm{ml}$ in culture medium. Suspended cells $(100 \mu \mathrm{l})$ were pipetted into each test well through one of the side ports of the Oris ${ }^{\mathrm{TM}}$ Cell Seeding Stopper. The seeded plate containing the Oris ${ }^{\mathrm{TM}}$ Cell Seeding Stoppers was incubated in a humidified chamber $\left(37^{\circ} \mathrm{C}, 5 \% \mathrm{CO}_{2}\right)$ for $24 \mathrm{~h}$ to permit cell attachment. Using the Stopper Tool, all the stoppers were removed. The media was removed gently and the wells washed with PBS to remove the unattached cells. After that, $100 \mu \mathrm{l}$ of FBS-free medium containing Calcein AM (final concentration $0.5 \mu \mathrm{g} / \mathrm{ml}$ ) was added, and the cells were incubated in a humidified chamber $\left(37^{\circ} \mathrm{C}, 0.5 \% \mathrm{CO}_{2}\right)$ for $40 \mathrm{~min}$. The images were captured under a fluorescence microscope (DM 3000B; Leica), and these data served as initial control. After fluorescence intensity examination, media were removed gently, and washed twice with PBS, and $100 \mu \mathrm{l}$ of medium containing vehicle, Bat or CLT was added, and the cells were incubated in a humidified chamber $\left(37^{\circ} \mathrm{C}, 0.5 \% \mathrm{CO}_{2}\right)$ for $24 \mathrm{~h}$. At the end of treatment, data were obtained as indicated above.

Cell immunofluorescence. E-cadherin and vimentin immunofluorescence in MDA-MB-468 and MDA-MB-231 cells were analyzed. Briefly, log-phase cells were seeded into wells of 8 -well-Chamber Slide system (Nunc ${ }^{\circledR}$ ). Cells were grown at $37^{\circ} \mathrm{C}$ in a humidified $\mathrm{CO}_{2}$ incubator until $50-70 \%$ confluence, and exposed to CLT and/or vehicle for $24 \mathrm{~h}$. After exposure, the chambers were gently removed, and slides were rinsed twice in PBS. Cells fixed by $4 \%$ paraformaldehyde solutions were blocked against non-specific antibody interactions with $5 \%(\mathrm{w} / \mathrm{v}) \mathrm{BSA}$ in TBST for $1 \mathrm{~h}$ at room temperature and incubated overnight at $4^{\circ} \mathrm{C}$ with anti-E-cadherin $(1: 100,3195)$ or vimentin antibody $(1: 100,3932)$ (both from Cell Signaling Technology). After washing in TBST, slides were incubated with a secondary antibody conjugated with Alexa Fluor 555 (4413, 1:500; Cell Signaling Technology) for $1 \mathrm{~h}$. Cells were then washed and incubated with ProLong Gold antifade reagent with DAPI (Invitrogen). Samples were examined under a fluorescent microscope (BX63; Olympus).
Protein extraction and western blot analysis. Cells were rinsed twice with PBS, and total proteins were extracted in $500 \mu 1$ lysis buffer. Aliquots of whole cell lysates were separated by $10 \%$ SDS-PAGE and then transferred to Hybond nitro blotting membranes. The membranes were blocked with 3\% BSA in Tris-buffered saline containing $0.5 \mathrm{ml} / 1$ Tween-20 and then incubated with primary antibodies against E-cadherin (3195; Cell Signaling Technology), N-cadherin (ab18203; Abcam), vimentin (3932; Cell Signaling Technology), cytokeratin 19 (ab15463), Snail (ab82846) and Slug (ab27568) (all from Abcam), Twist (sc-6070; Santa Cruz Biotechnology), Smad2 (ab55478; Abcam), phosphorSmad2/3 (sc-11769) and Runx2 (SC-10758) (both from Santa Cruz Biotechnology), phospho-Runx2 (AP3559a, Abgent), JNK1/2/3 (ab59227) and phospo-JNK1/2/3 (ab192200) (both from Abcam), ERK1/2 (sc-292838) and phosphor-ERK1/2 (sc-16982) (both from Santa Cruz Biotechnology), p38MAPK (9212) and phospho-p38MAPK (9211) (both from Cell Signaling Technology) followed by incubation with horseradish peroxidase (HRP)-conjugated secondary antibodies. Immunoreactive proteins were detected using an enhanced chemiluminescence kit (Millipore). $\beta$-actin (SC-130301; Santa Cruz Biotechnology) served as an internal control.

Data analysis. The data are presented as mean \pm SD and were analyzed with SPSS for Windows (13.0) software program (Chicago, IL, USA). Comparison among different groups was carried out by one-way analysis of variance (the one-way ANOVA). Differences between means were considered statistically significant at $\mathrm{P}<0.05$.

\section{Results}

CLT shows significant inhibition on TGF- $\beta 1$-mediated EMT in breast cancer cells. To understand the effects of CLT on EMT, the initial study was to check expression of E-cadherin in CLT-treated MDA-MB-231 and MDA-MB-468 cells. As shown in Fig. 2A, when MDA-MB-231 cells were incubated with TGF- $\beta 1$, the epithelial cell marker E-cadherin was significantly inhibited. After exposure to CLT solution at various concentrations for $24 \mathrm{~h}$, expression of this epithelial cell marker was increased in a dose-dependent manner. In addition, to verify the anti-EMT effects of CLT, another breast cancer cell line was studied, which has been demonstrated to be susceptible to undergo EMT changes under appropriate stimuli (18). Reduced E-cadherin was observed when MDA-MB-468 cells were incubated with TGF- $\beta 1$, and CLT increased the expression of this marker significantly (Fig. 2B).

EMT programs are associated classically with a set of change of cell-surface and cytoskeleton markers, which are orchestrated by a set of pleiotropically acting transcription factors. Thus, we next determined the effects of CLT on several prototypical epithelial and mesenchymal markers by western blotting assay, including cell surface molecular, cytoskeleton proteins and transcription factors. As shown in Fig. 3, CLT enhanced E-cadherin and CK19 expression in both MDA-MB-231 and MDA-MB-468 cells. In addition, CLT significantly inhibited expression of $\mathrm{N}$-cadherin and vimentin, which are mesenchymal markers and acquired during EMT. 
A MDA-MB-231
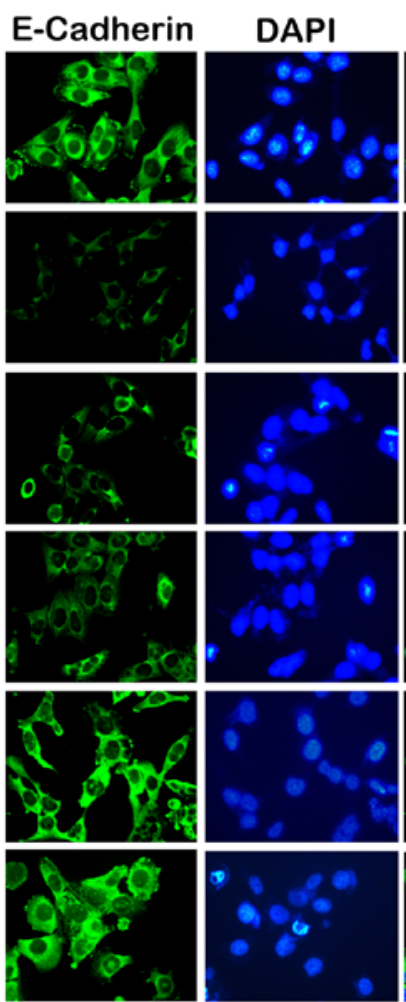

merged
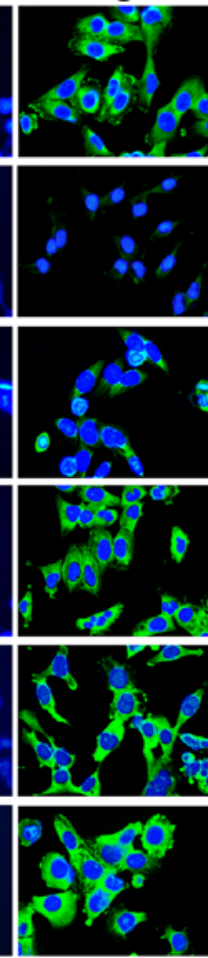

B

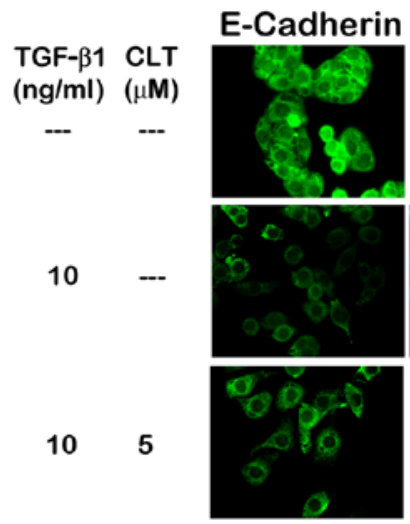

10

10
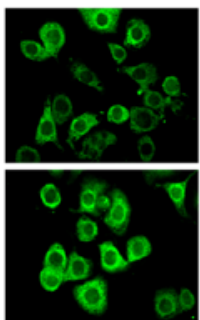

10

40
MDA-MB-468
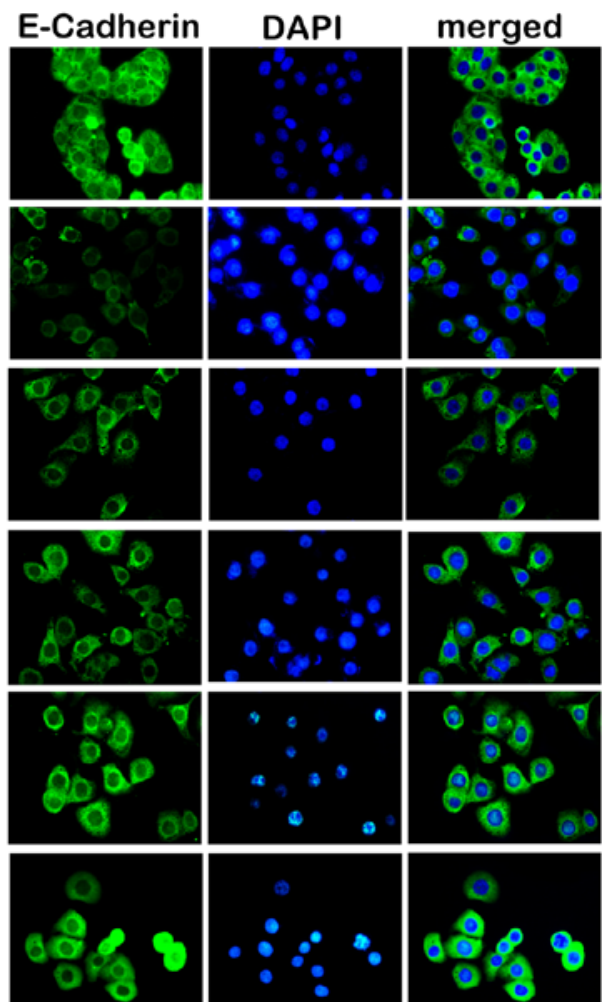

Figure 2. Effects of CLT on expression of E-cadherin in breast cancer cells. (A) Effects of CLT on E-cadherin in MDA-MB-231 cells. (B) Effects of CLT on E-cadherin in MDA-MB-468 cells. E-cadherin expression in breast cancer cells was analyzed by cell immunofluorescence assay. Vehicle (DMSO) was used as a CTRL.

Furthermore, we also found that expression of several transcription factors, Snail, Slug and Twist 1, was significantly blocked by CLT (Fig. 3) in a dose-dependent manner. These data demonstrated the effects of CLT on TGF- $\beta 1$-induced EMT in vitro.

CLT inhibits EMT of MDA-MB-468 tumors in vivo. According to the study by the Gilles group (18), MDA-MB-468 is an inducible model of EMT in vivo. To determine whether CLT impairs EMT in vivo, we established an orthotopic xenograft model by injection of MDA-MB-468 cells into the mammary fat pad in NOD/SCID mice following the method of Gilles et al. It was found that compared with vehicle-treated animals, CLT administered at $75 \mathrm{mg} / \mathrm{kg} /$ day caused very slight inhibition on primary tumor growth (Fig. 4), which is in agreement with our previous results. Next, the anti-EMT effects of CLT were evaluated by examining expression of two kinds of cytoskeleton proteins in tumor tissues by IHC. The most representative results are illustrated in Fig. 5 and semi-quantitative analysis in the tumor blocks is shown in Table I. It was found that CLT significantly enhanced CK19 expression, and the increased rate of CK19 is $126.62 \%$ compared with control (CTRL) when CLT was administered at $75 \mathrm{mg} / \mathrm{kg} / \mathrm{day}$. In addition, a $72.03 \%$ ( $\mathrm{P}<0.01$ compared with CTRL) and $88.73 \%$ ( $\mathrm{P}<0.01$ compared with CTRL) reduction of positive area of vimentin staining were observed when the nude mice were treated with 25 and $75 \mathrm{mg} / \mathrm{kg} /$ day CLT, respectively. These data suggested that CLT attenuated EMT in metastatic breast cancer, which may contribute to its anti-metastatic property.
Repression of TGF- $\beta$ signaling contributes to CLT-mediated EMT inhibition. Known for their ability to stimulate bone formation, TGF- $\beta$ signaling forms a vicious cycle in tumor progression and metastasis (19) and TGF- $\beta 1$ is regarded as the key growth factor involved in driving EMT (20). To determine whether CLT-mediated inhibition of EMT programming was through TGF- $\beta$ signaling pathway suppression, the effects of CLT on activity of TGF- $\beta$ signaling transducers were examined. The initial assay was to check the expression of Smad2 and phosph-Smad2/3, the TGF- $\beta$ selective Smad complex, by western blotting assay in MDA-MB-468 cells. Our data in Fig. 6 show that CLT significantly reduced phosph-Smad 2/3 expression in the presence of $10 \mathrm{ng} / \mathrm{ml}$ TGF- $\beta 1$, but there was no obvious change on expression of Smad2. Next, the effects of CLT on Smad-independent TGF- $\beta$ signaling and crosstalk with other pathway were also checked. It was found that phosphorylation of p38MAPK, ERK1/2 and JNK1/2/3 were decreased by CLT in a dose-dependent manner when MDA-MB-468 cells were co-incubated with $10 \mathrm{ng} / \mathrm{ml}$ TGF- $\beta 1$. Collectively, these findings indicated that CLT inhibited TGF- $\beta 1$-driven EMT programming by interfering with TGF- $\beta$ signaling in breast cancer cells.

CLT inhibits TGF- $\beta 1$ induced Runx2 phosphorylation in both MDA-MB-231 and MDA-MB-468 cells. In a previous study, we demonstrated that the activation of Runx2, a key transcription factor of skeletal formation, was abolished by CLT in metastatic breast cancer cells. To determine whether TGF- $\beta 1$ induces Runx 2 activation, and whether Runx 2 activation induced by TGF- $\beta 1$ is suppressed by CLT 

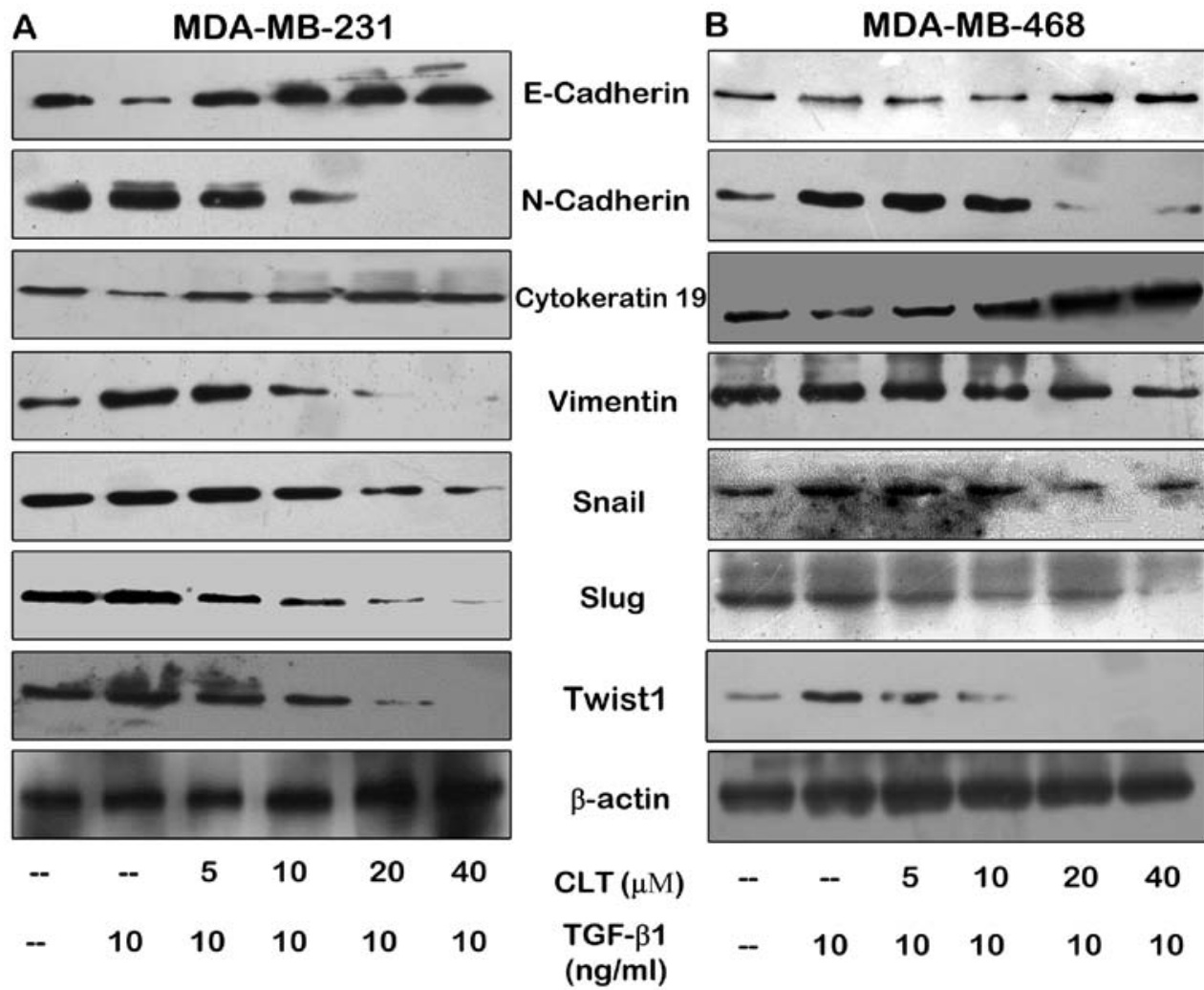

Figure 3. Effects of CLT on expression of EMT biomarkers in breast cancer cells. (A) Effects of CLT on EMT markers in MDA-MB-231 cells. (B) Effects of CLT on EMT markers in MDA-MB-468 cells. The expression of EMT marker in breast cancer cells were analyzed by cell immunofluorescence assay. Vehicle (DMSO) was used as a CTRL.
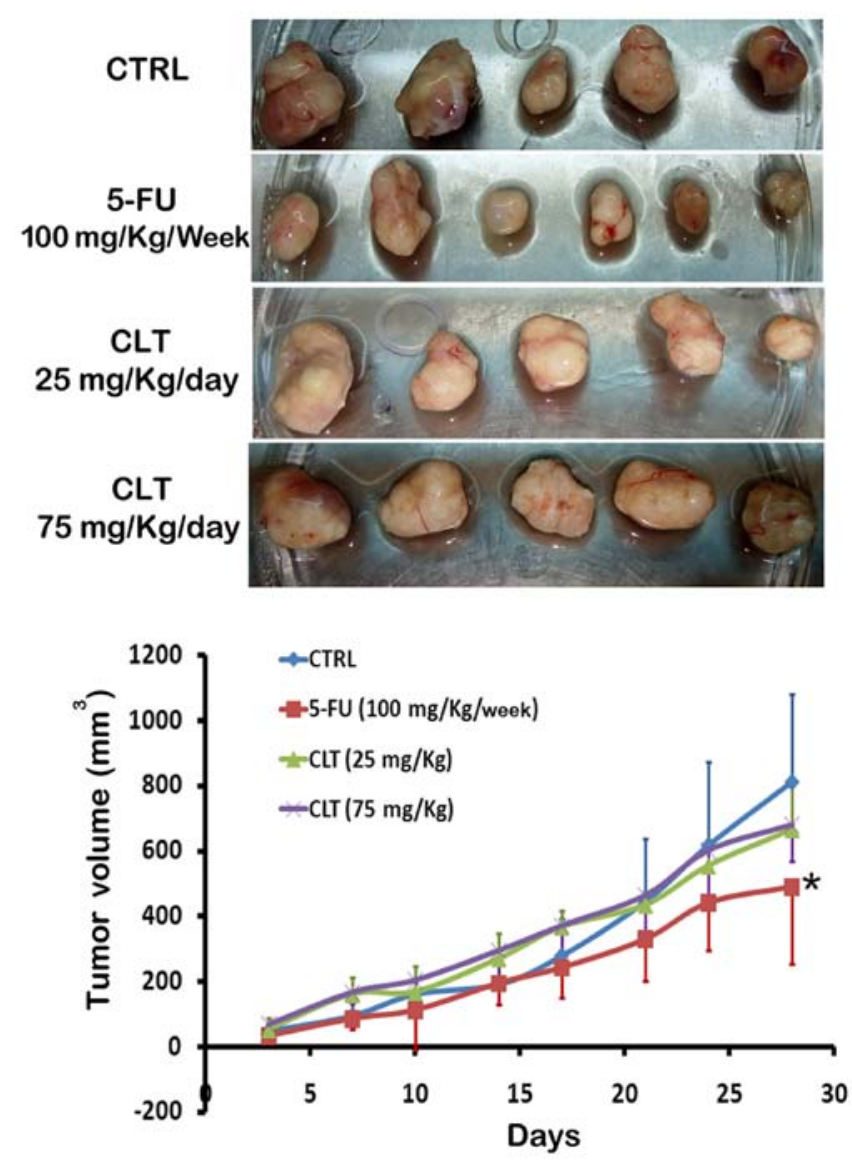

Figure 4. In vivo effects of CLT on MDA-MB-468 tumors growth. CLT slightly suppresses tumor growth in vivo, which is in agreement with our previous results. "P<0.05 compared with CTRL (control). 


\section{A Immunostaining for CK $19(\times 200)$}

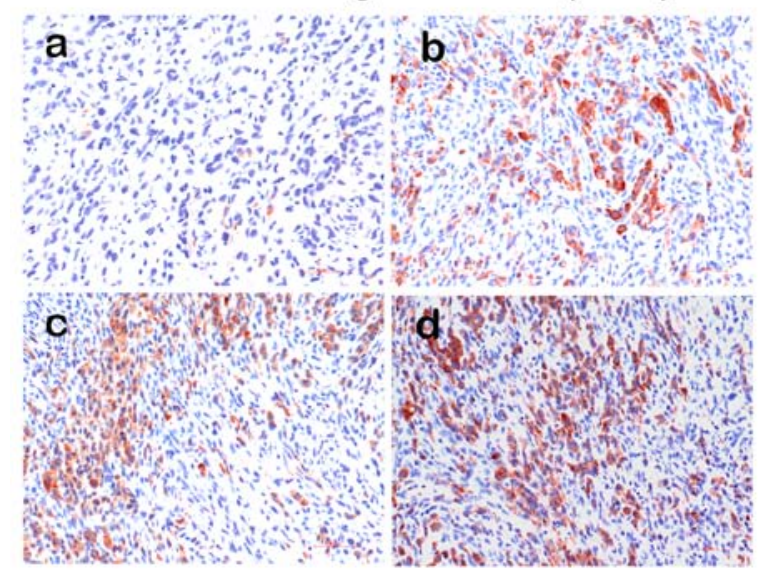

\section{B Immunostaining for Vimentin (x200)}
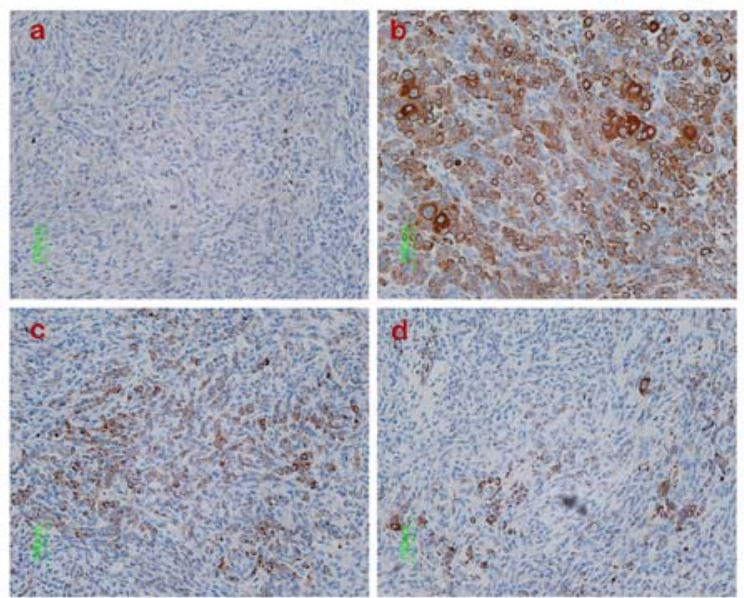

Figure 5. Effects of CLT on CK19 and vimentin in MDA-MB-468 tumor tissues. (A) Expression of CK19 in MDA-MB-468 tumor tissues. (B) Expression of vimentin in MDA-MB-468 tumor tissues. Expression of CK19 and vimentin in tumor tissues was detected by immunohistochemistry as described in Materials and methods section. The most representative results are illustrated in the figures. a, blank, no primary antibody incubated. b, CTRL; c, CLT (25 mg/kg); CLT $(75 \mathrm{mg} / \mathrm{kg}$.)

Table I. Semi-quantitative analysis of CK19 and vimentin in MDA-MB-468 tumor tissues.

\begin{tabular}{lccccc}
\hline & \multicolumn{2}{c}{ CK19 immunostaining } & & \multicolumn{2}{c}{ Vimentin immunostaining } \\
\cline { 2 - 2 } \cline { 5 - 6 } Group & Relative area $(\%)^{\mathrm{a}}$ & Increased rate $(\%)$ & & Relative area (\%) & Decreased rate (\%) \\
\hline Blank & $1.12 \pm 0.30^{\mathrm{b}}$ & - & & $0.47 \pm 0.15$ & - \\
CTRL & $21.30 \pm 2.56^{\mathrm{c}}$ & - & & $73.37 \pm 8.04^{\mathrm{c}}$ & - \\
CLT $25 \mathrm{mg} / \mathrm{kg}$ & $26.93 \pm 3.74$ & 26.43 & & $20.52 \pm 1.92^{\mathrm{d}}$ & 72.03 \\
CLT 75 mg/kg & $48.27 \pm 7.91^{\mathrm{d}}$ & 126.62 & & $8.27 \pm 1.23^{\mathrm{d}}$ & 88.73 \\
\hline
\end{tabular}

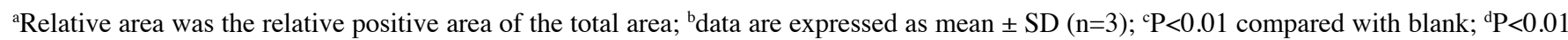
compared with control; significant difference was calculated by one-way ANOVA.

in metastatic breast cancer cells, we examined the effects of CLT on Runx2 and phosph-Runx2 expression when $10 \mathrm{ng} / \mathrm{ml}$ of TGF- $\beta 1$ was added in the culture system. The initial study was to evaluate expression of Runx2 and phosph-Runx2 in MDA-MB-231 and MDA-MB-468 cells in the presence and/ or the absence of TGF- $\beta 1$. As shown in Fig. 7A, Runx2 was expressed in both MDA-MB-231 and MDA-MB-468 cells in the absence of TGF- $\beta 1$. When TGF- $\beta 1$ was added in the culture system, there was no significant change of expression of Runx 2 in either of the breast cancer lines. However, the situation of p-Runx 2 expression was different. When there was TGF- $\beta 1$ in the culture system, phosphorylation of Runx2 was stimulated drastically in both cell lines. Next, effects of CLT on Runx2 and p-Runx2 expression were evaluated in the presence of $10 \mathrm{ng} / \mathrm{ml}$ of TGF- $\beta 1$. As shown in Fig. 7B, expression of p-Runx 2 induced by TGF- $\beta 1$ in MDA-MB-231 


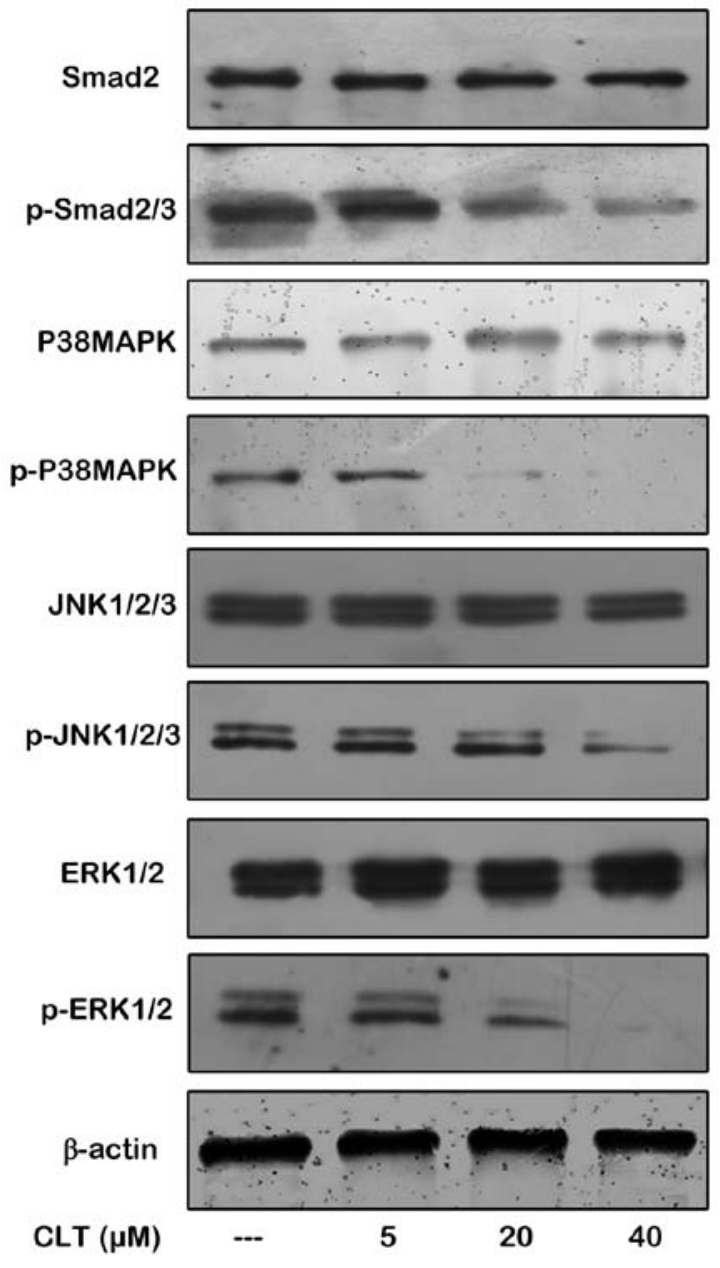

Figure 6. CLT suppression of TGF- $\beta$ signaling transducer activity in MDAMB-468 cells.

and MDA-MB-468 cells was decreased, respectively, by CLT in a dose-dependent manner, but there was no effect on Runx2 expression compared to untreated control. These data indicated that in breast cancer cells, activation of Runx 2 stimulated by TGF- $\beta 1$ may be abolished by CLT.

CLT inhibits TGF- $\beta 1$-induced motility of metastatic breast cancer cells in vitro. Through EMT, epithelial-derived tumor cells acquire migratory and invasive capacity. The migration and invasion of epithelial-derived carcinoma cells are the essential steps to form metastatic foci. Thus, we next determined the effects of CLT on invasion and migration induced by TGF- $\beta 1$. As shown in Fig. 8A, CLT blocked TGF- $\beta 1$-induced invasion of breast cancer cells through re-constitutive basement membrane significantly, when the cells were incubated with 10 and $40 \mu \mathrm{M}$ CLT for $14 \mathrm{~h}$ in the presence of $10 \mathrm{ng} / \mathrm{ml}$ TGF- $\beta 1$. We next evaluated the effects of CLT on cell migration with the Oris ${ }^{\mathrm{TM}}$ cell migration system. Here, we also found that migration of both breast cancer cell types was significantly blocked by CLT (Fig. 8B) in a dose-dependent manner.

\section{Discussion}

The EMT is a biologic process that allows a polarized epithelial cell, which normally interacts with basement membrane

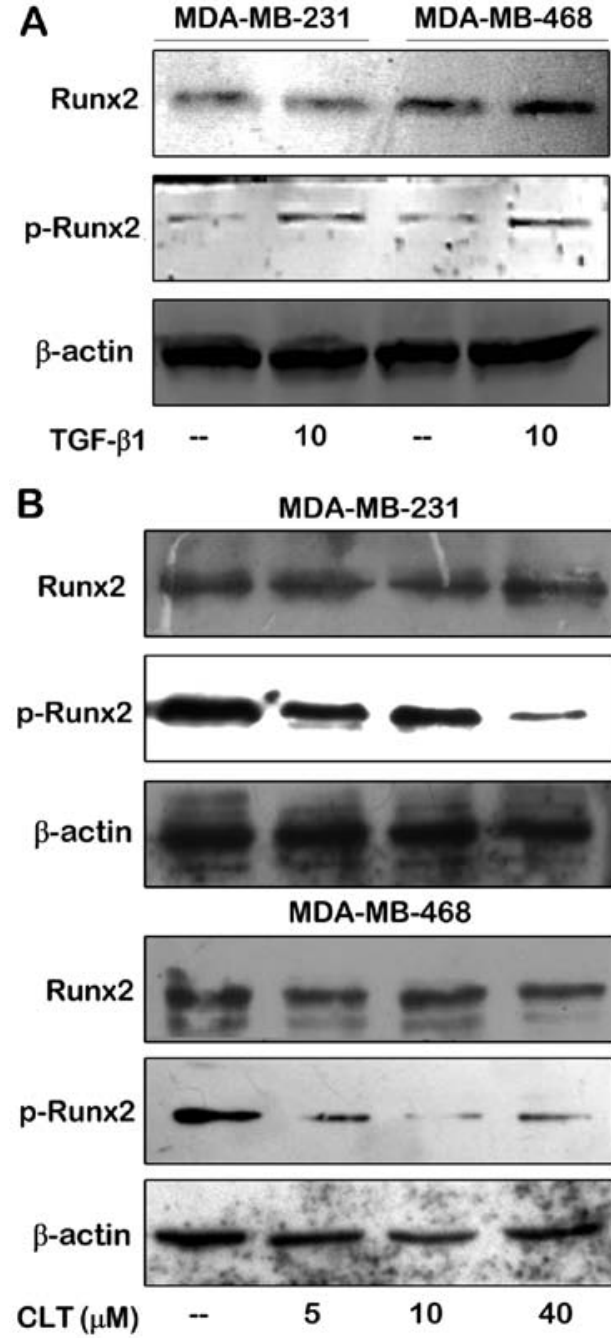

Figure 7. Effects of CLT on activation of Runx 2 transcription factor induced by TGF- $\beta 1$ in MDA-MB-231 and MDA-MB-468 cells. (A) Expression of Runx2 and p-Runx2 in MDA-MB-231 and MDA-MB-468 cells. (B) CLT suppressed phosphorylation of Runx 2 in both breast cancer cell lines.

via its basal surface, to undergo multiple biochemical changes that enable it to assume a mesenchymal cell phenotype, which includes enhanced migratory capacity, invasiveness, elevated resistance to apoptosis, and greatly increased production of extracellular matrix (ECM) components (21). For the progression of human carcinoma, EMT is also seen in a set of carcinoma cells undergoing phenotypic conversion for invasion and metastasis. Through EMT, epithelial-derived tumor cells promote genetic alterations and acquire a mesenchymal cell phenotype, including down-modulating cell-cell adhesion structures, altering their polarity, reorganizing their cytoskeleton, and becoming isolated, motile, and resistant to anoikis (21). Previously, we revealed that CLT exhibited anti-metastatic effects in metastatic breast cancer cells, and these effects may be mediated by inhibition of MMPs via downregulation of the transcriptional activity of Runx2 (14). Runx2 is a key transcription factor associated with osteoblast differentiation and breast cancer metastasis (15). Extensive evidence shows that there is a close association between abnormal activation of Runx 2 and matrix degradation, colonizing in bone marrow, angiogenesis and EMT. In addition, it is reported that Runx2 is 


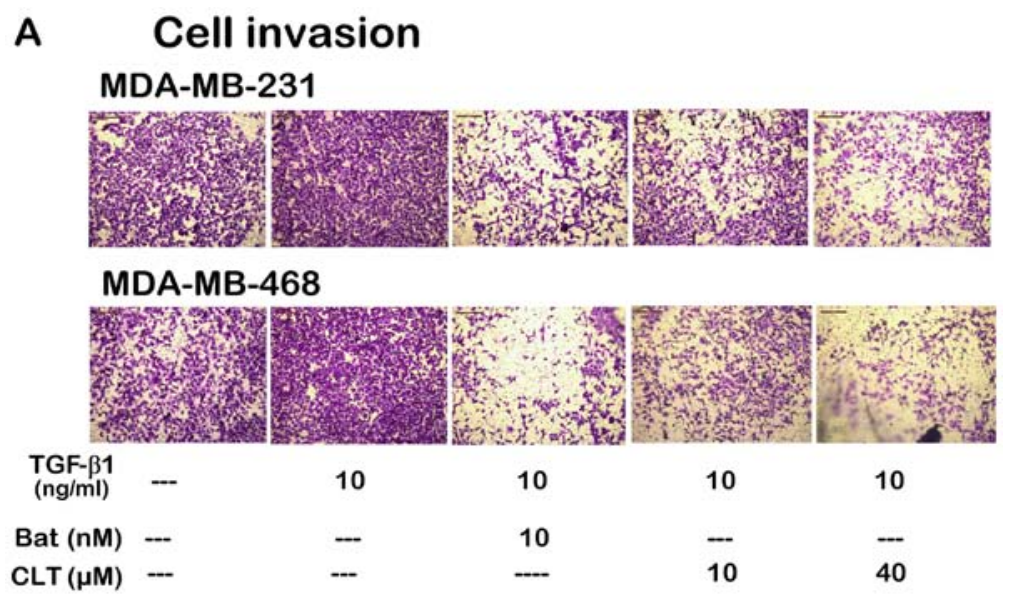

\section{B Cell migration MDA-MB-231}
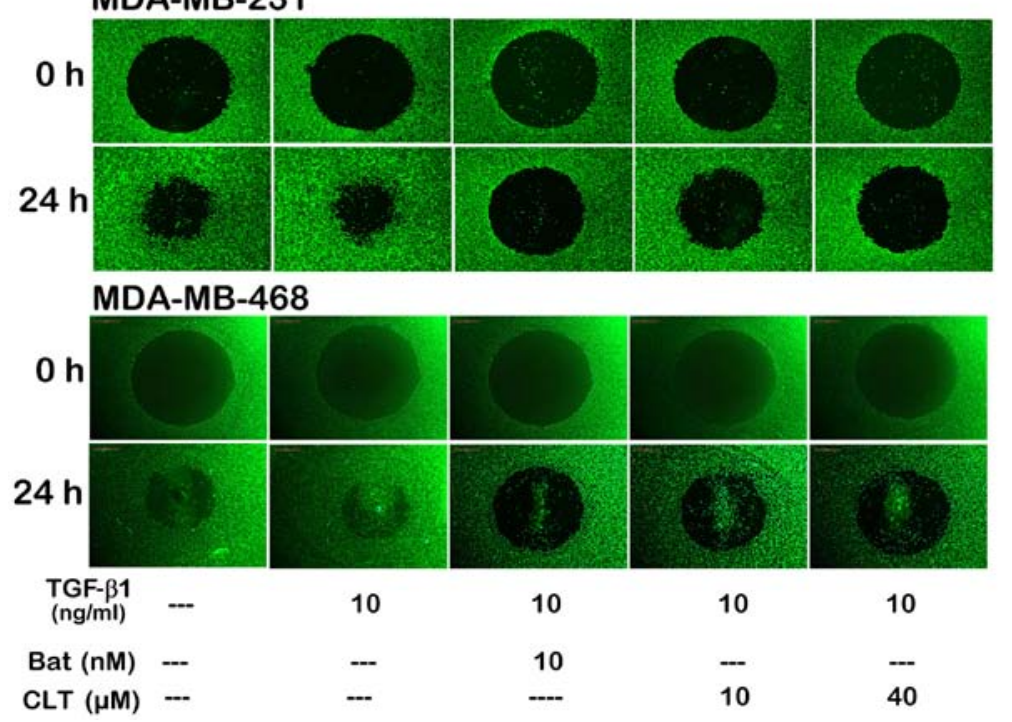

Figure 8. Effects of CLT on invasion and migration of breast cancer cells. (A) CLT inhibited the invasion of MDA-MB-231 and MDA-MB-468 cells. (B) Effects of CLT on in vitro migration of MDA-MB-231 and MDA-MB-468 cells. In vitro invasion and migration assay was carried out as described in Materials and methods section. Vehicle (DMSO) was used as a CTRL and Bat served as the positive CTRL. Each assay was performed in triplicate.

a common target of TGF- $\beta$ signaling (16). By activating Smad proteins and other signal transduction pathways to stimulate expression of Runx2, TGF- $\beta 1$ stimulate osteoblast-specific gene expression (17). Led by these concepts, we sought to find out whether the EMT-involved mechanisms and related signaling contribute to the anti-metastatic effects of CLT in breast cancer.

EMT describes a series of rapid changes in cellular phenotype, including epithelial cells loosening cell-cell adhesion structures, modulating their polarity and rearranging their cytoskeleton (22). A set of molecules is involved in transition, some of which are acquired, and some are attenuated. Recently, most of them have been used as biomarkers of EMT to monitor EMT. These molecules include expression of specific cell-surface proteins, reorganization and expression of cytoskeletal proteins, production of ECM-degrading enzymes, and changes in the expression of specific microRNAs (23). cadherins (named for 'calcium-dependent adhesion') are a class of type-1 transmembrane proteins, which constitute a large family of cell surface proteins including cadherins, protocadherins, desmogleins, and desmocollins. They play important roles in cell adhesion, forming adherens junctions to bind cells within tissues (24). E-cadherin, one of cadherins expressed on the epithelial cell surface, is decreased during EMT in cancer progression, but N-cadherin, a mesenchymal cadherin, is increased during EMT. This kind of changes in the level of expression of E-cadherins and N-cadherin, so-called cadherin switches, has been increasingly used to monitor progress of EMT during cancer progression (25). Vimentin, which is a type III intermediate filament protein expressed in mesenchymal cells, is acquired during EMT. This cytoskeleton protein is commonly used as a biomarker to identify cells undergoing EMT in cancers (26). In the present study, these typical biomarkers were studied to check the effects of CLT on EMT. We demonstrated using cell immunofluorescence assay that CLT significantly inhibited TGF- $\beta 1$-driven cadherin switch in both MDA-MB-231 and MDA-MB-468 cells. By western blot assay, we verified that CLT enhanced expression of epithelial markers in both breast cancer cells, such as epithelial cell surface marker E-cadherin and cytoskeleton marker CK19, and that CLT also attenuated expression of mesenchymal markers, such as mesenchymal 
cell surface marker $\mathrm{N}$-cadherin and cytoskeleton marker vimentin. Moreover, in vivo study demonstrated that CLT increased CK19 expression and decreased vimentin expression in MDA-MB-468 tumors significantly, which means CLT blocked EMT programming of MDA-MB-468 tumors. Taken together, our data indicated that CLT arrests programming of EMT, which may contribute to its anti-metastatic properties in breast cancer.

In order to initiate an EMT and enable it to reach completion, a number of distinct transcription factors are engaged in this process. Snail transcription factors are one prominent example of a common trans-acting regulatory factor binding to the promoter of various EMT-associated genes. The Snail family of zinc-finger transcription factors consist of Snaill (Snail), Snail2 (Slug) and Snail3 (Smuc), and the members are the most widely recognized as key regulators of various aspects of the EMT phenotype, such as increased expression of mesenchymal cells (fibronectin and vitronectin), decreased expression of various epithelial markers (E-cadherin and cytokeratins) (27). Twist proteins are highly conserved basic helix-loop-helix (bHLH) transcription factors that have important regulatory functions during lineage determination and cell differentiation. Numerous studies have demonstrated that this master regulator of embryonic morphogenesis plays a crucial role in metastasis (28). According to Yang et al, Twist can act independently of Snail to repress E-cadherin and to upregulate fibronectin and $\mathrm{N}$-cadherin in the development of metastatic cancer cells by EMT (29). Thus, we sought to determine whether CLT inhibits expression of EMT-associated transcription factors. Our data showed that CLT reduced TGF- $\beta 1$-driven expression of Snail, Slug and Twist1 in both breast cancer cell types. Collectively, these data indicated that CLT-induced decrease of the EMT-associated transcription factors may result in change of expression of cell surface and cytoskeleton proteins driven by TGF- $\beta 1$, leading to EMT blockade in breast cancer.

Several signaling pathways are involved in transcription program switching of EMT, such as TGF- $\beta$, BMP, Wnt/ $\beta$-catenin, Notch, Hedgehog signaling, and TGF- $\beta$ signaling pathway is the most well-characterized pathway that is known to induce EMT (6,30-32). By activating Smad-dependent signaling, TGF- $\beta$ promotes the transcription of key genes associated with EMT. Complexes of R-Smads are able to bind directly to the promoter of snail to induce its transcription, and can form complexes with Snail to suppress the expression of genes encoding E-cadherin and occludin $(33,34)$. Other factors directly influenced by the binding of R-Smads include the ZEB transcription factors and the high mobility group factor HGMA2, which also regulates the expression of snail, snai2, and twist genes $(33,35)$. Besides acting through Smad pathway, TGF- $\beta$ also activates Smad-independent pathway during EMT. These pathways have non-transcriptional roles in EMT, including dissolution of epithelial junctions, cytoskeletal reorganization and motility, and translational control. They also target Smads and thus help define their functions, while controling the expression and activation of transcription factors, with which Smad complexes cooperate in the control of gene expression (6). The JNK and p38MAPK cascades can be activated by TGF- $\beta$-activated kinase 1 (TAK1) which is involved in the induction of snail transcrip- tion in mammary epithelial cells in a Smad-independent manner during EMT (36). Early evidence indicated that MAPK kinase (MEK)-ERK signaling is activated by TGF- $\beta$, which is consistent with the observation of induction of EMT transcription factors, such as Twistl (37). As TGF- $\beta$ and the TGF- $\beta$-related proteins have been shown as major inducers of EMT, we hypothesized that CLT-induced EMT inhibition was through suppression of TGF- $\beta$ signal pathway. This hypothesis was supported by our data from western blotting. Our data showed that CLT significantly inhibited TGF- $\beta 1$ induced activation of Smad-dependent and -independent signaling in MDA-MB-468 cells. These findings indicated that CLT inhibited TGF- $\beta 1$-driven EMT programming by interfering with TGF- $\beta$ signaling in breast cancer cells.

Runx2 (also named PEBP2 $\alpha$ A/AML3/Cbfa1) is a transcription factor of the runx gene family, encoding proteins homologous to Drosophila Runt (38). Originally, this transcription factor was thought to contribute to skeletal formation (39), but extensive research indicates the role of Runx 2 in metastatic growth of breast cancer cells. In breast cancer cells, several genes correlated with the occurrence of skeletal metastasis regulated by Runx2, such as $b s p, o p n$, $m m p-9, m m p-13$, and vegf (40). Therefore, Runx2 is reported to be a 'master' transcription factor of metastatic growth of breast cancer cells, including homing to bone, induction of high bone turnover, angiogenesis and tissue invasion (41). Studies from the signal pathway in osteoblast differentiation and cancer metastasis reveal that this transcription factor is a common target of the TGF- $\beta$ /BMP signal pathway. By activating Smad proteins and other signal transduction pathways to stimulate expression of Runx $2, \mathrm{TGF}-\beta$ stimulated osteoblast-specific gene expression $(16,17)$. More recently, studies have demonstrated that Runx 2 is a crucial regulator of EMT. Chimge et al demonstrated that Slug is a Runx 2 target required for mediating its pro-metastatic property (42). By transient interference RNA assay, Niu et al demonstrated that EMT-associated transcription factors, such as Snail, Slug and Twist1, are target of Runx 2 in thyroid carcinoma cells (43). Thus, Runx2 is a crucial transducer of EMT and metastasis, which is regulated by TGF- $\beta$ /BMP signal and regulates the EMT phenotype. Previously, we showed that downregulation of Runx 2 transcription activity may be involved in inhibition of MMP expression induced by CLT. Thus, we investigated whether Runx 2 is activated by TGF- $\beta$ and CLT blocks activation of Runx 2 in breast cancer cells. Our data from western blot assay showed that TGF- $\beta 1$ significantly stimulated Runx2 phosphorylation in both MDA-MB-231 and MDA-MB-468 cells, and CLT inhibited TGF- $\beta 1$-induced p-Runx 2 expression, indicating that the transcriptional ability of Runx 2 is impaired by CLT in breast cancer cells.

Collectively, our present study demonstrated that CLT inhibited programming of EMT in vitro and in vivo, resulting in inhibition of motility of metastatic breast cancer cells. The inhibitory effect of CLT was due to its ability to inhibit TGF- $\beta$ signaling and Runx2 phosphorylation.

\section{Acknowledgements}

This study was supported by grants from the National Natural Science Foundation of China (grant nos. 81560639, 81160530, 
and 81260656), the Key Research Project from the Ministry of Education of China (grant no. 211091), and the Natural Science Foundation of Jiangxi Province (grant no. 2010GQY0147).

\section{References}

1. Siegel R, Ma J, Zou Z and Jemal A: Cancer statistics, 2014. CA Cancer J Clin 64: 9-29, 2014.

2. Liu S, Goldstein RH, Scepansky EM and Rosenblatt M: Inhibition of rho-associated kinase signaling prevents breast cancer metastasis to human bone. Cancer Res 69: 8742-8751, 2009.

3. Thiery JP, Acloque H, Huang RY and Nieto MA: Epithelial-mesenchymal transitions in development and disease. Cell 139: 871-890, 2009.

4. Yilmaz M and Christofori G: EMT, the cytoskeleton, and cancer cell invasion. Cancer Metastasis Rev 28: 15-33, 2009.

5. Son $\mathrm{H}$ and Moon A: Epithelial-mesenchymal transition and cell invasion. Toxicol Res 26: 245-252, 2010.

6. Gonzalez DM and Medici D: Signaling mechanisms of the epithelial-mesenchymal transition. Sci Signal 7: re8, 2014.

7. Chen Q, He H, Li P, Zhu J and Xiong M: Identification and quantification of atractylenolide I and atractylenolide III in Rhizoma Atractylodes macrocephala by liquid chromatography-ion trap mass spectrometry. Biomed Chromatogr 27: 699-707, 2013.

8. Kang TH, Bang JY, Kim MH, Kang IC, Kim HM and Jeong HJ: Atractylenolide III, a sesquiterpenoid, induces apoptosis in human lung carcinoma A549 cells via mitochondria-mediated death pathway. Food Chem Toxicol 49: 514-519, 2011.

9. Zhang NN, Park DK and Park HJ: The inhibitory activity of atractylenolide III, a sesquiterpenoid, on IgE-mediated mast cell activation and passive cutaneous anaphylaxis (PCA). J Ethnopharmacol 145: 278-285, 2013.

10. Li CQ, He LC and Jin JQ: Atractylenolide I and atractylenolide III inhibit lipopolysaccharide-induced TNF-alpha and NO production in macrophages. Phytother Res 21: 347-353, 2007.

11. Liu C, Zhao H, Ji ZH and Yu XY: Neuroprotection of atractylenolide III from Atractylodis macrocephalae against glutamate-induced neuronal apoptosis via inhibiting caspase signaling pathway. Neurochem Res 39: 1753-1758, 2014

12. Wang $\mathrm{KT}$, Chen $\mathrm{LG}, \mathrm{Wu} \mathrm{CH}$, Chang $\mathrm{CC}$ and Wang $\mathrm{CC}$ : Gastroprotective activity of atractylenolide III from Atractylodes ovata on ethanol-induced gastric ulcer in vitro and in vivo. $\mathrm{J}$ Pharm Pharmacol 62: 381-388, 2010.

13. Kang TH, Han NR, Kim HM and Jeong HJ: Blockade of IL-6 secretion pathway by the sesquiterpenoid atractylenolide III. J Nat Prod 74: 223-227, 2011.

14. Wang W, Chen B, Zou R, Tu X, Tan S, Lu H, Liu Z and $\mathrm{Fu} J$ : Codonolactone, a sesquiterpene lactone isolated from Chloranthus henryi Hemsl, inhibits breast cancer cell invasion, migration and metastasis by downregulating the transcriptional activity of Runx2. Int J Oncol 45: 1891-1900, 2014.

15. Ferrari N, McDonald L, Morris JS, Cameron ER and Blyth K: RUNX2 in mammary gland development and breast cancer. J Cell Physiol 228: 1137-1142, 2013.

16. Lee KS, Kim HJ, Li QL, Chi XZ, Ueta C, Komori T, Wozney JM, Kim EG, Choi JY, Ryoo HM, et al: Runx2 is a common target of transforming growth factor beta1 and bone morphogenetic protein 2, and cooperation between Runx 2 and Smad5 induces osteoblast-specific gene expression in the pluripotent mesenchymal precursor cell line C2C12. Mol Cell Biol 20: 8783-8792, 2000.

17. Phimphilai M, Zhao Z, Boules $\mathrm{H}$, Roca $\mathrm{H}$ and Franceschi RT: BMP signaling is required for RUNX2-dependent induction of the osteoblast phenotype. J Bone Miner Res 21: 637-646, 2006.

18. Bonnomet A, Syne L, Brysse A, Feyereisen E, Thompson EW, Noël A, Foidart JM, Birembaut P, Polette M and Gilles C: A dynamic in vivo model of epithelial-to-mesenchymal transitions in circulating tumor cells and metastases of breast cancer. Oncogene 31: 3741-3753, 2012.

19. Zhang Q, Yu N and Lee C: Vicious cycle of TGF- $\beta$ signaling in tumor progression and metastasis. Am J Clin Exp Urol 2: 149-155, 2014.

20. Borthwick LA, Gardner A, De Soyza A, Mann DA and Fisher AJ: Transforming growth factor- $\beta 1$ (TGF- $\beta 1)$ driven epithelial to mesenchymal transition (EMT) is accentuated by tumour necrosis factor $\alpha(\mathrm{TNF} \alpha)$ via crosstalk between the SMAD and NF- $\kappa$ B pathways. Cancer Microenviron 5: 45-57, 2012.
21. Savagner P: The epithelial-mesenchymal transition (EMT) phenomenon. Ann Oncol 21 (Suppl 7): vii89-vii92, 2010.

22. Lamouille S, Xu J and Derynck R: Molecular mechanisms of epithelial-mesenchymal transition. Nat Rev Mol Cell Biol 15: 178-196, 2014.

23. Zeisberg M and Neilson EG: Biomarkers for epithelial-mesenchymal transitions. J Clin Invest 119: 1429-1437, 2009.

24. Angst BD, Marcozzi C and Magee AI: The cadherin superfamily: Diversity in form and function. J Cell Sci 114: 629-641, 2001

25. Gheldof A and Berx G: Cadherins and epithelial-to-mesenchymal transition. Prog Mol Biol Transl Sci 116: 317-336, 2013.

26. Eriksson JE, Dechat T, Grin B, Helfand B, Mendez M, Pallari HM and Goldman RD: Introducing intermediate filaments: From discovery to disease. J Clin Invest 119: 1763-1771, 2009.

27. Wang Y, Shi J, Chai K, Ying X and Zhou BP: The role of Snail in EMT and tumorigenesis. Curr Cancer Drug Targets 13: 963-972, 2013.

28. Khan MA, Chen HC, Zhang D and Fu J: Twist: A molecular target in cancer therapeutics. Tumour Biol 34: 2497-2506, 2013.

29. Yang Z, Zhang X, Gang H, Li X, Li Z, Wang T, Han J, Luo T, Wen $\mathrm{F}$ and $\mathrm{Wu} X$ : Up-regulation of gastric cancer cell invasion by Twist is accompanied by $\mathrm{N}$-cadherin and fibronectin expression. Biochem Biophys Res Commun 358: 925-930, 2007.

30. Derynck R, Muthusamy BP and Saeteurn KY: Signaling pathway cooperation in TGF- $\beta$-induced epithelial-mesenchymal transition. Curr Opin Cell Biol 31: 56-66, 2014.

31. Thomson S, Petti F, Sujka-Kwok I, Mercado P, Bean J, Monaghan M, Seymour SL, Argast GM, Epstein DM and Haley JD: A systems view of epithelial-mesenchymal transition signaling states. Clin Exp Metastasis 28: 137-155, 2011.

32. Xu J, Lamouille S and Derynck R: TGF-beta-induced epithelial to mesenchymal transition. Cell Res 19: 156-172, 2009.

33. Peinado H, Quintanilla $\mathrm{M}$ and Cano A: Transforming growth factor beta-1 induces snail transcription factor in epithelial cell lines: Mechanisms for epithelial mesenchymal transitions. J Biol Chem 278: 21113-21123, 2003.

34. Vincent T, Neve EP, Johnson JR, Kukalev A, Rojo F, Albanell J, Pietras K, Virtanen I, Philipson L, Leopold PL, et al: A SNAIL1-SMAD3/4 transcriptional repressor complex promotes TGF-beta mediated epithelial-mesenchymal transition. Nat Cell Biol 11: 943-950, 2009.

35. Thuault S, Tan EJ, Peinado H, Cano A, Heldin $\mathrm{CH}$ and Moustakas A: HMGA2 and Smads co-regulate SNAIL1 expression during induction of epithelial-to-mesenchymal transition. J Biol Chem 283: 33437-33446, 2008.

36. Yamashita M, Fatyol K, Jin C, Wang X, Liu Z and Zhang YE: TRAF6 mediates Smad-independent activation of JNK and p38 by TGF-beta. Mol Cell 31: 918-924, 2008.

37. Xie L,Law BK, Chytil AM, Brown KA, Aakre ME and Moses HL: Activation of the Erk pathway is required for TGF-beta1-induced EMT in vitro. Neoplasia 6: 603-610, 2004.

38. Stein GS, Lian JB, van Wijnen AJ, Stein JL, Montecino M, Javed A, Zaidi SK, Young DW, Choi JY and Pockwinse SM: Runx 2 control of organization, assembly and activity of the regulatory machinery for skeletal gene expression. Oncogene 23: 4315-4329, 2004

39. Komori T: Runx2, a multifunctional transcription factor in skeletal development. J Cell Biochem 87: 1-8, 2002.

40. Inman CK and Shore P: The osteoblast transcription factor Runx 2 is expressed in mammary epithelial cells and mediates osteopontin expression. J Biol Chem 278: 48684-48689, 2003.

41. Gupta GP and Massagué J: Cancer metastasis: Building a framework. Cell 127: 679-695, 2006.

42. Chimge NO, Baniwal SK, Little GH, Chen YB, Kahn M, Tripathy D, Borok Z and Frenkel B: Regulation of breast cancer metastasis by Runx 2 and estrogen signaling: The role of SNAI2. Breast Cancer Res 13: R127, 2011.

43. Niu DF, Kondo T, Nakazawa T, Oishi N, Kawasaki T, Mochizuki K, Yamane T and Katoh R: Transcription factor Runx2 is a regulator of epithelial-mesenchymal transition and invasion in thyroid carcinomas. Lab Invest 92: 1181-1190, 2012. 